\title{
Molecular Studies on Extra-Pulmonary Tuberculosis among Patients Attending Aminu Kano Teaching Hospital, Kano, Kano State, Nigeria.
}

\author{
1,3* Mohammad A.B., ${ }^{2}$ Zakari F., ${ }^{2}$ Yusuf A.S., \\ ${ }^{2}$ Sani N.M., ${ }^{3}$ Gwarzo A.M., 5 lliya S., \\ ${ }^{4}$ Gwarzo, F.S. and ${ }^{6}$ Mukhtar M.D. \\ ${ }^{1 *}$ North West Zonal Tuberculosis Reference Laboratory, \\ Department of Medical Microbiology, \\ Aminu Kano Teaching Hospital, Kano, Nigeria. \\ ${ }^{2}$ Department of Microbiology and Biotechnology, \\ Federal University Dutse, \\ Jigawa State, Nigeria. \\ ${ }^{3}$ Department of Medical Microbiology and Parasitology, \\ Faculty of Basic Clinical Sciences, \\ College of Medicine and Allied Medical Sciences, \\ Federal University Dutse. \\ Jigawa State, Nigeria
}

${ }^{4}$ Kano State College of Education and Preliminary Studies, Kano State, Nigeria.

${ }^{5}$ Depertment of Biological Sciences, School of Pure and Applied Sciences, Mount Kenya University Thika, Kiambu County, Kenya.

${ }^{6}$ Department of Microbiology, Faculty of Life Science, Bayero University Kano, Nigeria.

Email: abmkyarama@gmail.com

\begin{abstract}
In 2010 World Health Organization reported that, one third of the World population had tuberculosis, and it is among the infectious disease with significant morbidity and mortality. Mycobacterium tuberculosis is a successful pathogen that has evolved several mechanisms to manipulate the host immune response. The purpose of this study was to determine the incidence of extra-pulmonary TB in patient that visited DOTs clinic, Aminu Kano Teaching Hospital, Kano Nigeria for treatment. A prospective cross-sectional laboratory based study that involved the use of questionnaire and consent/assent form prior to sample collection. Extra pulmonary tuberculosis samples were tested by MTB/RIF assay using GeneXpert. Descriptive statistics was used to analyzed the data obtained and results were given in charts and tables. Out of 71 samples analyzed along with
\end{abstract}


positive control (H37RV) and molecular grade water as a negative control, $3(4.2 \%)$ samples had extra-pulmonary TB, among which $2(2.8 \%)$ were from urine samples and 1 (1.4\%) from acetic fluid. This study shows the existence of extra-pulmonary tuberculosis in the study area; and the tuberculosis was sensitive Mycobacterium tuberculosis. It is therefore important to make screening of patients with sign and symptoms of extra pulmonary tuberculosis using Xpert MTB/RIF assay as regular method for extra pulmonary tuberculosis diagnosis. This will be achieved through enlightenment of the clinicians in our health facilities, our States and local governments should strengthen the laboratory capacity for diagnosis and make the services available and reachable to the patients who need them.

Keywords: Mycobacterium tuberculosis, Polymerase Chain Reaction, Extra-Pulmonary Tuberculosis, Prevalence.

\section{INTRODUCTION}

Tuberculosis (TB) continues to be a serious public health concern, with 8.6 million new infections, 0.8 million extrapulmonary tuberculosis (EPTB) cases, and 1.3 million deaths worldwide (Boehme et al., 2010). EPTB is a type of tuberculosis that affects parts of the body other than the lungs. It can affect almost any region of the body, but the lymph nodes and pleura are the most common sites (Al-Hajoj et al., 2013). Although active pulmonary tuberculosis is conveyed by coughing droplets, extrapulmonary tuberculosis is caused by haematogenous spread from an initial lung infection and is not infectious (Agresti et al., 1998). Extrapulmonary tuberculosis can occur alone or in conjunction with pulmonary tuberculosis (Al-Muhsen, 2010). Extra-pulmonary TB is especially common in people living with HIV as well as in children (Agarwal et al., 2005). Therefore, it is much more common in countries with a high HIV prevalence and in countries where a lot of children are infected with TB (Alonzo et al., 1999).

In many countries, including Nigeria, extrapulmonary tuberculosis, as well as the growth of multidrug-resistant tuberculosis (MDRTB), is a public health concern (Salami and Katibi 2006). Over a billion people around the world have latent tuberculosis (Range et al., 2016). WHO reported in 2012 that the incidence of extrapulmonary tuberculosis was 0.31 percent, reflecting a 50\% rise in incidence rates from 2011 and a 10\% fatality rate (Al-Otaibi et al., 2010). Drug-resistant tuberculosis was found to be present in 4.7 percent of follow-up tuberculosis patients in Kano State, Nigeria (Mohammad et al., 2017). According to the Nigerian Federal Ministry of Health, the country has an annual incidence of roughly 399,000 cases of tuberculosis in all forms, with a $10 \%$ prevalence rate, making it the fourth-highest TB burden in the world and the greatest in Africa (Awoyemi et al., 2002). When infected persons cough, spit, talk, or sneeze, tuberculosis spreads via the air. Every year, an untreated person with active tuberculosis can infect 10 to 15 people (WHO, 2011). Tuberculosis normally kills a person by progressively forming holes in the lungs, which then spread to other body organs, resulting in extrapulmonary tuberculosis (Alonzo and Pepe, 1999). People who are infected with Mycobacterium tuberculosis have a $10 \%$ lifetime risk of contracting tuberculosis (Alonzo Pepe, 1999; FMOHN, 2016). People with weakened immune systems, such as those who have the human immunodeficiency virus (HIV), malnutrition, diabetes mellitus, or who smoke, are at a substantially increased risk of contracting tuberculosis (Agresti, 1998; Mohammad et al., 2021). The fatal synergies between tuberculosis and HIV, as well as the evolution of medication resistance, have made TB control more difficult (Al-Hajoj et al. 2013; FMOHN, 2016). In general, different countries have reported an increasing proportion of EPTB (FMOHN, 2016). According to reports, people living with HIV have a greater rate of extrapulmonary tuberculosis because their 
immune systems are unable to respond strongly to a TB infection and thus are unable to confine the TB bacilli (Bruchfeld et al., 2002).

Because of its poor infectious potential, EPTB receives less attention than pulmonary tuberculosis. However, because of the diagnostic difficulties as well as the potential for high morbidity and death, it is regarded as a serious clinical condition (Alonzo and Pepe, 1999; Bruchfeld, 2002; Alrabiaah et al., 2012). A recent study from Brazil found that a lack of diagnostic capabilities is a critical factor in late EPTB diagnosis and treatment (Boehme et al. 2010; Bruchfeld et al. 2002; Alrabiaah et al. 2012). According to studies, one-third of the world's population is infected with tuberculosis, and $10 \%$ of those infected acquire tuberculosis during their lifetime. As a result, early detection of active tuberculosis remains tricky (Chijioke and Aderibigbe, 1998; Mohammad et al. 2020). Extrapulmonary tuberculosis diagnosis remains difficult in developing nations with limited resources, such as Nigeria. Furthermore, there are no data on extrapulmonary tuberculosis (TB) in the research area (Awoyemi et al., 2003; Onipede, 1999; Pai et al., 2004). Despite the rise in frequency and death rates, identifying extrapulmonary tuberculosis using traditional methods remains a challenge since it takes time and has low sensitivity and specificity when compared to molecular methods (Salami and Katibi, 2006; Pai et al., 2004). To reach a probable turnaround time of two hours, a molecular technique (using Genexpert) can be employed to identify sensitive Mycobacterium tuberculosis and treatment resistance (Range et al., 2016; Asghar et al., 2004). The WHO endorsed the Xpert MTB/RIF assay method as an alternative for sputum smear microscopy in December 2010. (FMOHN, 2016). The time it takes to identify Mycobacteria has been reduced by employing the GeneXpert assay, and it should be encouraged to be used as a tool for early EPTB diagnosis (Agresti, 1999; Bruchfeld et al., 2002; Mohammad et al.2020). The goal of this study was to find out how common extrapulmonary tuberculosis is among patients at Kano's Aminu Kano Teaching Hospital.

\section{MATERIALS AND METHODS}

\section{Study area and sample collection}

The study was carried out in Aminu Kano Teaching Hospital, Kano, Nigeria, which is one of Nigeria's most populous states, with over 15 million citizens. It is the most populous city in Northern Nigeria, and it is situated in the northwestern part of the country. The state has 44 local government areas, with the majority of population being Hausa/ Fulani. It contains a total of 1,339 health-care institutions (Public and Private). In Aminu Kano Teaching Hospital, there are four referral hospitals (including the University Teaching Hospital and the National Orthopedic Hospital), 379 DOTS centers, 84 microscopy sites (84), nineteen (19) GeneXpert machines distributed across the state, one MDR ward, and a North-West Zonal Tuberculosis Reference Laboratory. The hospital, which is located in the Tarauni local government area of Kano city, is the state's largest tertiary hospital (NTBLCP, 2014). The state borders Kaduna State to the south, Jigawa State to the west, and Bauchi State to the east, and has numerous wards, clinics, and theatres, including HIV, DOTS, ICU, isolation units, and theatres. This is a six-month cross-sectional prospective laboratory study of agreed and assented patients attending specialities and DOTS clinics at Aminu Kano Teaching Hospital in Kano, Nigeria, who displayed signs and symptoms of extrapulmonary tuberculosis. The study was approved by the ethics committee at Aminu Kano Teaching Hospital in Kano, Nigeria (certificate number: NHREC/21/08/2008/AKTH/EC/2546), and it was carried out in conformity with the Helsinki Declaration. A questionnaire was used to 
collect socio-demographic information, as well as other characteristics linked to extrapulmonary tuberculosis and the pattern of extrapulmonary tuberculosis presentation.

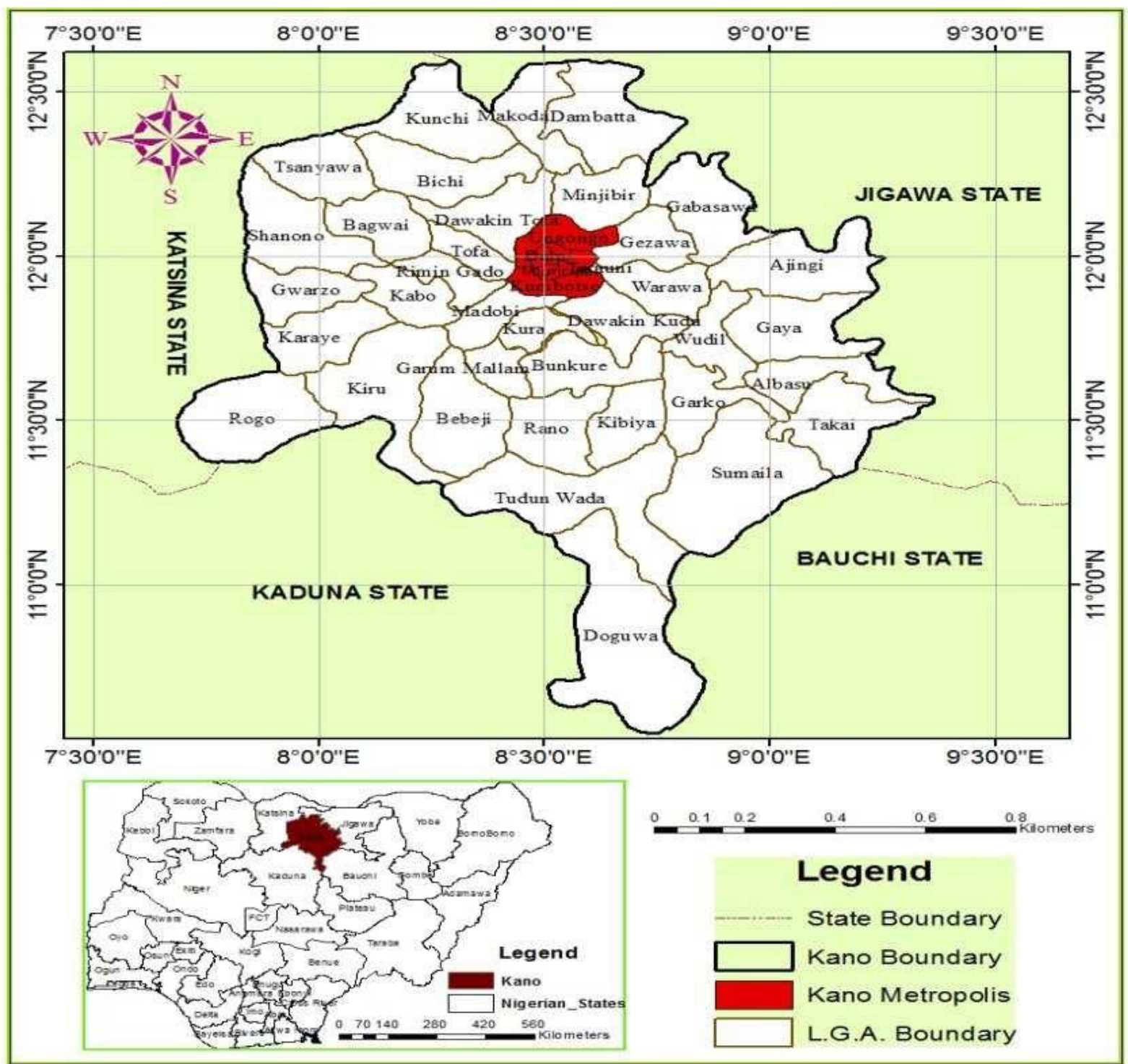

Figure 1: Map of Kano State

The sample size in this study was obtained using Cochrane's (1963) and Glenn's (1963) formula (2010). [z2 p (1- p)] $=\mathrm{n}$ [d2] [d2] [d2] [d2] [d2 Where $\mathrm{n}$ is the number of samples. $\mathrm{p}=$ prevalence $=4.92 \mathrm{z}=$ statistical level of confidence at 95 percent $=1.96 \mathrm{z}=$ statistical level of confidence at 95 percent $=1.96(0.0492) \mathrm{d}=$ a 5 -percentage-point margin of error $(0.05)$ $[(1.96) 2 \times 0.0492(1-0.0492)] \mathrm{n}=[(1.96) 2 \times 0.0492(1-0.0492)][(0.05) 2]=71[(0.05) 2]=71$ $[(0.05) 2]=71[(0.05)$ As a result, seventy-one (71) samples were obtained under aseptic conditions from suspected patients with extrapulmonary tuberculosis signs and symptoms who signed and consented or assented to participate in the study. Urine samples were collected in a clean gallon for 24 hours; samples were allowed to settle, the supernatant was decanted, and deposits were centrifuged using a chilled centrifuge (eppendorf centrifuge 5810R), stomach lavage, cerebral fluid, and ascetic fluid. The MTB/RIF assay was used to examine the deposits. 


\section{MTB/RIF ASSAY (PCR TEST)}

2 millilitres $(\mathrm{ml})$ of sample diluent were applied immediately to 1 millilitre $(\mathrm{ml})$ of sample (ratio of 2:1). The mixture was incubated for 15 minutes at room temperature, then votexed and re-incubated for 5 minutes at room temperature. $2 \mathrm{~mL}$ of the mixture was pipetted and dispensed into the cartridge using a sterile pasture pipette. Cartridges were identified on the left side with a sample identification number or by affixing a pre-labeled paper. The sterile transfer pipette was not touched. The bulb-end of the pipette was wrapped around the front. The liquefied sample was drawn into the sterile transfer pipette provided in the Xpert/Rif kit (Cepheid, Sunnyvale, California, USA) until the pipette's meniscus was above the minimum threshold $(2 \mathrm{ml})$. The pipette was inserted into the paper/plastic lid with care. The cartridge cover was closed after the transfer pipette was placed into a biohazard waste bin. The lid snaps were firmly placed and inserted onto the GeneXpert machine (Cepheid, Sunnyvale, California, USA) for 1 hour 55 minutes of running time. Only computer results were automatically printed.

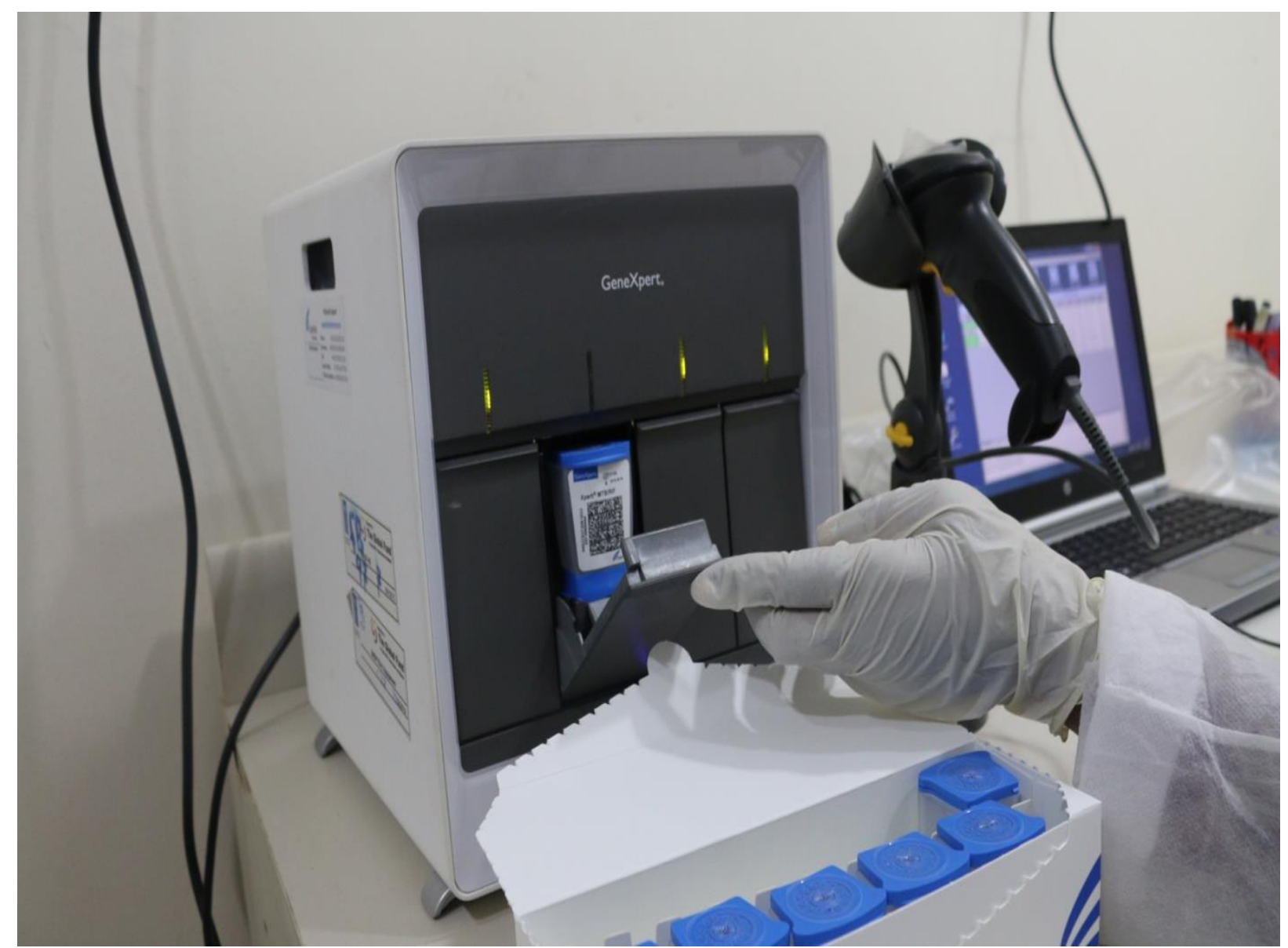

Figure 2: GeneXpert Machine

\section{RESULTS}

The socio-demographic characteristics of the TB patients in the study were acquired. More over $40 \%$ of the 71 patients in the study were between the ages of 21 and 40, with the majority of them being men ( 60.6 percent ). A tertiary education was held by 33.8 percent of the patients, a secondary education by 31.0 percent, and no formal education by 21.1 percent. In addition, 56.3 percent were married, 9.9\% widowed, 23.9 percent were single, and $9.9 \%$ were divorced (Table 1 ). 
Table 1: Socio-Demographic Characteristics of Studied Participants

\begin{tabular}{llll}
\hline Characteristics & Males & Females & Frequency (\%) \\
\hline Age & & & \\
$\leq 20$ & $2(2.8)$ & $1(1.4)$ & $3(4.2)$ \\
$21-30$ & $19(26.8)$ & $5(5.0)$ & $24(33.8)$ \\
$31-40$ & $10(14.1)$ & $10(14.1)$ & $20(28.2)$ \\
$41-50$ & $8(11.3)$ & $6(8.5)$ & $14(19.8)$ \\
$51-60$ & $5(7.0)$ & $2(2.8)$ & $7(9.8)$ \\
$>60$ & $1(1.4)$ & $2(2.8)$ & $3(4.2)$ \\
Marital status & & & \\
Single & $13(18.3)$ & $4(5.6)$ & $17(23.9)$ \\
Married & $25(35.2)$ & $15(21.1)$ & $70(56.3)$ \\
Widowed & $4(5.6)$ & $3(4.2)$ & $7(9.9)$ \\
Divorced & $3(4.2)$ & $4(5.6)$ & \\
& $3(4.2)$ & & $15(21.1)$ \\
Educational status & & & $10(14.1)$ \\
Non-formal & $6(12.7)$ & $9(12.7)$ & $22(31.0)$ \\
Primary & $3(4.2)$ & $7(9.9)$ & $24(33.8)$ \\
Secondary & $16(22.5)$ & $6(8.5)$ & \\
Tertiary & $20(28.2)$ & $4(5.6)$ & $25(35.2)$ \\
Occupation & & & $7(9.9)$ \\
Civil servants & $23(32.4)$ & $2(2.8)$ & $16(22.5)$ \\
Farmers & $5(7.0)$ & $2(2.8)$ & $10(14.1)$ \\
Business men/women & $10(14.1)$ & $6(8.5)$ & $10(14.1)$ \\
House wives & $0(0.0)$ & $10(14.1)$ & $3(4.20)$ \\
Students & $5(7.0)$ & $5(7.0)$ & \\
Unemployed & $2(2.8)$ & $1(1.4)$ & \\
& & & \\
& & &
\end{tabular}

\section{EXTRA-PULMONARY TUBERCULOSIS}

Drug-resistant tuberculosis (DR-TB) and sensitive MTB are two types of extrapulmonary tuberculosis. Patients with sensitive MTB were discovered in this investigation (Table 2).

Table 2: Number of cases of MTB segregated by sex

\begin{tabular}{llll}
\hline Gender & Number of samples screened & Indeterminate & MTB Detected \\
\hline Female & 26 & 0 & 1 \\
Male & 45 & 0 & 2 \\
Total & $\mathbf{7 1}$ & $\mathbf{0}$ & $\mathbf{3}$ \\
\hline
\end{tabular}

Note: $\mathrm{MTB}=$ Mycobacterium tuberculosis 
Factors associated with extra pulmonary tuberculosis among studied participants

\begin{tabular}{|c|c|c|c|}
\hline Factors & $\begin{array}{l}\text { Males } \\
(\%)\end{array}$ & $\begin{array}{l}\text { Females } \\
(\%)\end{array}$ & $\begin{array}{l}\text { Frequency } \\
(\%)\end{array}$ \\
\hline \multicolumn{4}{|l|}{ HIV Status } \\
\hline HIV Positive & $0(0.00)$ & $0(0.00)$ & 0 \\
\hline HIV Negative & $45(63.4)$ & $26(36.6)$ & $71(100)$ \\
\hline Total & $45(3.5)$ & $26(36.6)$ & $71(100)$ \\
\hline \multicolumn{4}{|c|}{ Contact with TB Patients } \\
\hline Yes & $2(2.8)$ & $1(1.4)$ & $3(4.2)$ \\
\hline No & $43(60.6)$ & $25(38.0)$ & $68(95.8)$ \\
\hline Total & $45(63.4)$ & $26(36.6)$ & $71(100)$ \\
\hline \multicolumn{4}{|c|}{ History of Diabetes } \\
\hline Yes & $0(0.0)$ & $0(0)$ & $0(0)$ \\
\hline No & $43(60.6)$ & $28(39.4)$ & $71(100)$ \\
\hline Total & $43(60.6)$ & $28(39.4)$ & $71(100)$ \\
\hline \multicolumn{4}{|c|}{ History of Smoking } \\
\hline Yes & $6(8.5)$ & $0(0)$ & 65 (91.6) \\
\hline No & $39(54.9)$ & $32(45.1)$ & $71(100)$ \\
\hline Total & 45 (63.4) & $26(36.6)$ & 71 (100) \\
\hline \multicolumn{4}{|c|}{ History of Alcohol Consumption } \\
\hline Yes & $2(2.8)$ & $0(0.0)$ & $2(0.0)$ \\
\hline No & $43(60.6)$ & $26(36.6)$ & $71(100)$ \\
\hline Total & 45 (63.4) & $26(36.6)$ & $71(100)$ \\
\hline
\end{tabular}

HIV: Human Immunodeficiency Virus

The investigation found three positive instances of EPTB, two of which were found in urine (urinary TB) and one in ascetic fluid. Extrapulmonary specimens account for $10 \%$ of tuberculosis cases in South East Nigeria, according to WHO (2011). Furthermore, urogenital tuberculosis accounts for 30 to $40 \%$ of all extrapulmonary tuberculosis in the region (WHO, 2011; Otu et al., 2013). It is known to be a secondary manifestation of the disease, as it developed from pulmonary tuberculosis like most other kinds of extrapulmonary tuberculosis (Mohammad et al., 2017). After disseminating from the latent stage, the miliary tuberculosis develops and spreads to various areas of the body. Males had a prevalence of 2.8 percent in this study, while females had a prevalence of 1.4 percent, which is lower than the 9.5 percent prevalence reported in Ilorin North Central, Nigeria (Chijioke, 1998), albeit this could be due to the small sample size employed in the current study. Furthermore, in the early 1980s, a 14 percent prevalence of extrapulmonary tuberculosis was observed in Lagos, South Western Nigeria, which is greater than the current study's findings (Chijioke, 1998; Mohammad et al., 2017). Despite the fact that there have been fewer studies on extrapulmonary tuberculosis than pulmonary tuberculosis due to the small number of patients and short duration of the studies, a more comprehensive and well-coordinated country-wide study on renal involvement in active EPTB in Nigeria is recommended (AlHajoj, 2013).

According to a study conducted by Awoyemi (2002) in Ibadan, Nigeria, the sensitivity of GeneXpert as a diagnostic tool is determined by its detection limit (Agresti and Coull, 1998). MTB was detected in the MTB/RIF assay at $131 \mathrm{CFU} / \mathrm{ml}$ of the material (Barnes, 1997). It was reported that the detection of leftover DNA from dead bacterial cells may have improved GeneXpert's sensitivity, giving it an advantage over regular culture and microscopy (WHO, 2011). According to a study conducted in the United States, the 
GeneXpert assay has higher sensitivity in the detection of MTB and Rif resistant MTB from urine samples (Asghar et al., 2004; Alrabiaah et al., 2012; Kruijshaar et al., 2006). Overexposure to the extreme alkaline environment of tubercle bacilli in urine samples, as a result of the same digested condition used in the treatment procedure for both sputum and urine samples, was responsible for inactivating a percentage of MTB, resulting in the low recovery rate by conventional methods, according to WHO (2011). (WHO, 2011). This procedure's hypothesis appears to be appropriate for sputum but overly stringent for pee (Onipede et al., 1999; Pai et al., 2004). As a result, one major explanation for our findings is that more research is needed to determine the best digestion condition for urine samples and other extrapulmonary tuberculosis materials. Despite having a superior technology for detecting extrapulmonary tuberculosis from urine samples, the main disadvantage of this assay is its high cost when compared to AFB smear microscopy. Furthermore, the rapid detection of extrapulmonary tuberculosis in this investigation supports the utility of the GeneXpert MTB/MTBRIF assay for the diagnosis of suspected EPTB patients; however, due of the small sample size, this conclusion should be interpreted with care. Nonetheless, the presence of extrapulmonary tuberculosis in the study area, with a prevalence of 4.2 percent, has drawn attention to the need for more research. For the use of the GeneXpert MTB/RIF assay in the diagnosis of suspected patients with extrapulmonary tuberculosis, the National Tuberculosis and Leprosy Control Programme and Non-Governmental Organizations supporting TB activities must intensify awareness campaigns to all medical and health workers, as well as the general public.

\section{CONCLUSION}

With the current conditions in Sub-Saharan Africa and much of the developing world, and the disease's slightly altered characteristics in our environment, diagnosing extrapulmonary tuberculosis using traditional methods may be problematic. As a result, patients with extrapulmonary tuberculosis symptoms should be investigated using the PCR MTB/RIF Assay, and all suspected EPTB patients should have access to these services so that appropriate treatment can be given to them right away, preventing the amplification of resistance in Mycobacterium tuberculosis.

ACKNOWLEDGEMENTS: All authors acknowledged the management of Aminu Kano Teaching Hospital, Kano, and Research and ethics committee of the hospital for giving us ethical approval; members of staff North West Zonal Tuberculosis Reference Laboratory Aminu Kano Teaching Hospital, Kano for all the contributions they rendered during the study.

\section{REFERENCES}

Agarwal SP, Chauhan LS. Tuberculosis control in India. Directorate General of Health Services/Ministry of Health and Family Welfare, New Delhi, India, 2005.

Agresti A, Coull B. Approximate is better than "exact" for interval estimation of binomial proportions. Am. Stat. 1998; 52:119-126.

Al-Hajoj S, Varghese B, Shoukri MM, Al-Omari R, Al-Herbwai M. Epidemiology of antituberculosis drug resistance in Saudi Arabia: findings of the first national survey. Antimicrob Agents Chemother. 2013; 57: 2161-2166. doi: 10.1128/AAC.02403-12 PMID: 23459478.

Alonzo TA, Pepe MS. Using a combination of reference tests to assess the accuracy of a new diagnostic test. Stat Med. 1999; 18:2987-3003. 
Alonzo T A, Pepe MS. Using a combination of reference tests to assess the accuracy of a new diagnostic test. Stat Med. 1999; 18:2987-3003.

Al-Otaibi F, El Hazmi MM. Extra-pulmonary tuberculosis in Saudi Arabia. Indian J Pathol Microbiol. 2010; 53: 227-231. doi: 10.4103/0377-4929.64327 PMID: 20551522.

Alrabiaah AA, Alsubaie SS, Bukhari EI, Gad A, Alzamel FA. Outbreak of BacilleCalmetteGuerin-related lymphadenitis in Saudi children at a university hospital after a change in the strain of vaccine. Ann Saudi Med. 2012; 32: 4-8. PMID: 22156633.

Asghar RJ, Pratt RH, Kammerer JS, Navin TR. Tuberculosis in South Asians living in the United States, 1993-2004. Arch Intern Med. 2008; 168: 936-942. doi: 10.1001/archinte.168.9.936 PMID: 18474757.

Awoyemi OB, Ige OM, Onadeko BO. Prevalence of active pulmonary tuberculosis in human immunodeficiency virus seropositive adult patients in University College Hospital, Ibadan, Nigeria. Afr J Med Med Sci. 2002; 31(4):329-32.

Barnes PF. Rapid diagnostic tests for tuberculosis: progress but no gold standard. Am. J. Respir. Crit. Care Med. 1997; 165:1497-1498.

Boehme C Catharina ${ }^{1}$, Pamela Nabeta, Doris Hillemann, Mark P Nicol, Shubhada Shenai, Fiorella Krapp, Jenny Allen, Rasim Tahirli, Robert Blakemore, Roxana Rustomjee, Ana Milovic, Martin Jones, Sean M O'Brien, David H Persing, Sabine Ruesch-Gerdes, Eduardo Gotuzzo, Camilla Rodrigues, David Alland, Mark D Perkins (2010): posting date. Rapid molecular detection of tuberculosis and rifampin resistance. N. Engl. J. Med. 2010; doi: 10.1056/NEJMoa0907847.

Bruchfeld J, Aderaye G, Palme IB, Bjorvatn B, Britton S, Feleke Y, et al. Evaluation of outpatients with suspected pulmonary tuberculosis in a high HIV prevalence setting in Ethiopia: clinical, diagnostic and epidemiological characteristics. Scand J Infect Dis. 2002; 34(5):331.

Cailhol J, Decludt B, Che D. Sociodemographic factors that contribute to the development of extrapulmonary tuberculosis were identified. J Clin Epidemiol 2005; 58: 1066-1071. PMID: 16168353.

Chijioke A and Aderibigbe A. Prevalence of renal tuberculosis among patients presenting with active pulmonary tuberculosis in Ilorin - Nigeria. African Journal of Nephrology. 1998; 2:34-38.

Federal ministry of Health Nigeria (FMOHN), report on Drug resistant TB survey. Global fund report on drug(s) resistant tuberculosis in Nigeria. 2016.

Kruijshaar ME, Abubakar I. Increase in extrapulmonary tuberculosis in England and Wales 1999-2006. Thorax. 2009; 64: 1090-1095. doi: 10.1136/thx.2009.118133 PMID: 19850965.

Mohammad AB, Iliyasu G, Habib GA. Prevalence and Genetic Determinant of Drugresistant Tuberculosis among Patients Completing Intensive Phase of Treatment in a Terciary Referal Centre in Nigeria: Int J Mycobateriol. 2017; Vol.6 issue 1. www.ijmyco.org

Mohammad, A. B., Usman, A. D., Aminu, A. I., Yakubu, A., Magashi, A. M., Mukhtar, M.D., Kawo, A.H.,Iliya, S., Hauwa, Y., Gwarzo, F.S., Abdulkadir, A., Sheshe, A.A. and Taura, D.W. Molecular diagnosis of Urinary Mycobacterium tuberculosis Among Patients Attending Urology Clinic in Aminu Kano Teaching Hospital, Kano, Nigeria. BJMLS, (2020) 5(2): 11 - 17 ISSN 2545 - 5672; eISSN 2635 - 3792

Mohammad, A.B., Ibrahim, A., Maifada, A.I., Balogun, M,S., Mohammed, Y., Fagge, H.S., Iliya, S., Aminu, A.I. and Mukhtar, M.D. Prevalence and Factors Associated with Rifampicin Resistant Pulmonary Tuberculosis among Presumptive Patients in Kano State, Nigeria Nigerian Journal of Microbiology, (2021) 35(1): - 5460 - 5466 
Onipede AO, Idigbe O, Ako-Nai AK, Omojola O, Oyelese AO, Aboderin AO. Seroprevalence of HIV antibodies in tuberculosis patients in Ile-Ife, Nigeria. East Afr Med J. 1999;76(3):127-32.

Otu A, Umoh V, Habib A, Ameh S, Lawson L, Ansa V. Drug resistance among pulmonary tuberculosis patients in Calabar, Nigeria. Pulm Med. 2013; 2013:235190.

Pai M, Flores, LL, Hubbard A, Riley LW, Colford JM. Nucleic acid amplification tests in the diagnosis of tuberculous pleuritis: a systematic review and meta-analysis. BMC Infect Dis. 2004; 4:6.

Range N, Magnussen P, Mugomela A, Malenganisho W, Changalucha J, Temu MM. (2016): HIV and parasitic co-infections in tuberculosis patients: a cross-sectional study in Mwanza, Tanzania.

Salami AK, Katibi IA. Human immunodeficiency virus associated tuberculosis: pattern and trend in the University of Ilorin Teaching Hospital. Afr J Med Med Sci. 2006.

World Health Organization (2011): Policy Statement: Automated real-time Nucleic Acid Amplification Technology for Rapid and Simultaneous Detection of Tuberculosis and Rifampicin Resistance: Xpert MTB/RIF System. Geneva, World Health Organization, 2011. 